\title{
RELACIÓN DEL ÍNDICE DE MASA CORPORAL (IMC) Y CIRCUNFERENCIA DE CINTURA (CC) CON GLUCOSA, COLESTEROL Y TRIGLICÉRIDOS EN ESTUDIANTES DE MEDICINA
}

\author{
RELATIONSHIP OF BODY MASS INDEX (BMI) AND WAIST \\ CIRCUMFERENCE (CC) WITH GLUCOSE, CHOLESTEROL AND \\ TRIGLYCERIDES IN MEDICAL STUDENTS
}

\author{
Carlos Alberto Chacón Zenteno ${ }^{1}$ \\ quimicocarloschacon@hotmail.com \\ Miguel Ángel Rodríguez Feliciano ${ }^{2}$ \\ mcsmarf@gmail.com
}

1 Universidad Pablo Guardado Chávez. Tuxtla Gutiérrez, México 
Para citar este artículo:

Chacón Z. Carlos, Gómez, J., Rodríguez M. (2020) Relación del Índice de Masa Corporal (IMC) y Circunferencia de Cintura (CC) con Glucosa, Colesterol y Triglicéridos en Estudiantes de Medicina. Espacio I+D, Innovación más Desarrollo. IX(23), 69-83. doi: http://dx.doi.org/10.31644/IMASD.23.2020.a05

\section{RESUMEN}

Se realizó un estudio para relacionar el índice de masa corporal (IMC) y circunferencia de cintura (CC) con la glucosa, colesterol y triglicéridos en 294 estudiantes con un promedio de 20 años inscritos en la licenciatura en Médico Cirujano de una escuela privada en Tuxtla Gutiérrez Chiapas en el año 2018. Se encontró que $36,4 \%$ de estudiantes presentó sobrepeso y 12,9 con obesidad tipo I. No se encontró diferencia estadísticamente significativa entre estas variables, sin embargo, se observó una tendencia al sobrepeso en estudiantes que tuvieron valores normales de glucosa (34\%), colesterol (45\%), triglicéridos (36\%), y con valores altos de estos parámetros 70\%, 48\% y 50\% respectivamente. Para CC tampoco se registró diferencia significativa entre estas variables, sin embargo, se encontró un riesgo de salud alto para enfermedades cardiovasculares en estudiantes con hiperglicemia (10\%), hipercolesterolemia (16\%) y con riesgo moderado con hipertrigliceridemia (40\%).

\section{Palabras Clave}

Hipercolesterolemia; hiperglicemia; hipertrigliceridemia; obesidad; sobrepeso 


\section{- Abstract-}

A study was conducted to relate the body mass index (вмг) and waist circumference (CC) with glucose, cholesterol and triglycerides in 294 students with an average of 20 years enrolled in the degree in Surgeon of a private school in Tuxtla Gutiérrez Chiapas in 2018. It was found that $36.4 \%$ of students were overweight and 12.9 with type I obesity. There was no statistically significant difference between these variables. However, a tendency to overweight was observed in students who had normal values of glucose $(34 \%)$, cholesterol $(45 \%)$, triglycerides $(36 \%)$, and with high values of these parameters $70 \%, 48 \%$ and $50 \%$ respectively . For Cc, there was also no significant difference between these variables, however, a high health risk was found for cardiovascular diseases in students with hyperglycemia (10\%), hypercholesterolemia (16\%) and with moderate risk with hypertriglyceridemia (40\%).

\section{Keywords}

Hypercholesterolemia; hyperglycemia; hypertriglyceridemia; obesity; overweight. 
L os indicadores antropométricos como el Índice de Masa Corporal (IMC) y el Índice de Circunferencia de Cintura (CC) se encuentran incrementados con la prevalencia de sobrepeso u obesidad y proporcionan la medida más útil para determinar estas variables en la población, pues es la misma para ambos sexos y para los adultos de todas las edades.

El sobrepeso y la obesidad constituyen un importante factor de riesgo de defunción, con una mortalidad de alrededor de 3 millones de adultos al año. De acuerdo con la Organización Mundial de la Salud (oms), la obesidad es una enfermedad crónica, caracterizada por el aumento de la grasa corporal, asociada a mayor riesgo para la salud. En 2018, más de 1900 millones de adultos de 18 o más años tenían sobrepeso, de los cuales, más de 650 millones eran obesos (oms, 2018).

De acuerdo con la Organización Panamericana de la Salud (ops), el sobrepeso y la obesidad van de la mano con diversas enfermedades crónicas e incapacitantes, entre ellas las más destacables son las enfermedades cardiovasculares, la hipertensión arterial, la diabetes mellitus no insulinodependiente, la osteoporosis y varios tipos de neoplasias malignas (Parreño y Gutiérrez, 2010).

Se sabe que la glucosa es un nutriente necesario para el organismo, pero el aumento progresivo de ésta puede originar diabetes. La diabetes se está convirtiendo en una epidemia mundial y relacionada con el rápido aumento del sobrepeso, la obesidad y la inactividad física. A escala mundial se calcula que 422 millones de adultos tenían diabetes en 2014. La diabetes causó 1,5 millones de muertes en 2012 y las elevaciones de la glucemia por encima de los valores ideales provocaron otros 2,2 millones de muertes por efecto de un aumento del riesgo de sufrir enfermedades cardiovasculares y de otro tipo (oms, 2016).

El colesterol y los triglicéridos son sustancias lipídicas importantes en nuestro organismo, pero en estos últimos años existe un aumento global de personas que poseen estos valores elevados, los cuales pueden causar diversas enfermedades no transmisibles como obesidad, enfermedades cardiovasculares, hipertensión arterial, diabetes mellitus, etc. Asimismo, estos valores elevados pueden reflejar la poca actividad física o los malos hábitos alimenticios (Parreño y Gutiérrez, 2010).

En este mismo sentido, se sabe que existe una importante correlación entre los niveles de colesterol y la morbimortalidad por enfermedad coronaria, en particular a partir de los $200 \mathrm{mg} / \mathrm{dl}$ de Colesterol total. Los niveles elevados de triglicéridos no son un factor de riesgo cardiovascular, pero sí constituyen un marcador de riesgo vascular cuando se asocian con otros factores de riesgos mayores, emergentes y vinculados a los hábitos de vida. (Organización Panamericana de la Salud. Situación de las enfermedades no transmisibles en el Perú. Ministerio de Salud; Lima, 2003 en Parreño y Gutiérrez, 2010). 
En México actualmente alrededor de siete de cada 10 adultos y uno de cada tres niños de 5 a 19 años presentan peso excesivo (sobrepeso u obesidad), y la diabetes se está convirtiendo en una epidemia mundial relacionada con el rápido aumento del sobrepeso, la obesidad y la inactividad física. En el mundo hay más de 347 millones de personas con diabetes (Rivera et al., 2013).

En Chiapas, el problema de obesidad y sobrepeso no es diferente al del país, en la Encuesta nacional de salud y nutrición (2018), el sobrepeso y la obesidad en niños menores de 5 años registró una prevalencia del $47.1 \%$ Estatal y de $28 \%$ en zona urbana y del $19.1 \%$ en la zona rural. Asimismo, estudios realizados por la Secretaría de Salud del Estado de Chiapas, registraron que los adolescentes de 12 a 19 años presentaron un porcentaje de sobrepeso y obesidad de $28.9 \%$, y para los adultos mayores de 20 años fue de $70.9 \%$ para hombre y $60.6 \%$ para mujeres (Instituto Nacional de Salud Pública, 2013).

Un estudio realizado en estudiantes universitarios en 2015, reportó una prevalencia de $15,0 \%$ de sobrepeso y el $2,3 \%$ obesidad. Los estudiantes pertenecientes al grupo masculino, mayores de 23 años, y los que fuman actualmente, presentaron los mayores valores de prevalencias de sobrepeso y obesidad $(22,0 \% ; 23,7 \% ; 25,5 \%)$ respectivamente. Además, en términos de salud, los alumnos entre 18 y 25 años presentaron un índice de masa corporal superior a $25 \mathrm{~kg} / \mathrm{m} 2$, y que es el sexo masculino el que se asocia como factor predisponente para presentar sobrepeso u obesidad (Tuta-García et al., 2015).

Considerando lo anterior, este trabajo de investigación tiene como objetivo principal relacionar el índice de masa corporal y la circunferencia de cintura, con la glucosa, el colesterol y los triglicéridos en estudiantes universitarios de la carrera de medicina, ya que, estudios realizados por la Secretaría de Salud del Estado de Chiapas registraron que los adolescentes de 12 a 19 años presentan un porcentaje de sobrepeso y obesidad de $28.9 \%$, y para los adultos mayores de 20 años fue de $70.9 \%$ para hombre y $60.6 \%$ para mujeres. La tendencia de esta prevalencia no es muy alentadora, pues va al alza conforme aumenta la edad (Instituto Nacional de Salud Pública, 2013).

\section{MATERIALES Y MÉTODOS}

En el presente estudio se trabajó con una población total de 294 estudiantes (175 mujeres y 119 hombres); ello porque, a decir de (Núñez, 2014), se trata del "conjunto de todos los elementos que forman parte del espacio territorial al que pertenece el problema de investigación”, en este caso, al conjunto de todos los alumnos matriculados de primero a octavo semestre de la licenciatura de médico cirujano de una escuela del sector privado y que accedieron a ser parte del estudio mediante consentimiento informado por escrito, de acuerdo con lo establecido a la Ley General de Salud y al 
Acta de Helsinki para investigaciones médicas en seres humanos, así como las buenas prácticas clínicas.

Las técnicas para la recolección de datos que se aplicaron en la investigación son las siguientes: Para evaluar el Índice de Masa Corporal (IMC) en los estudiantes, se utilizó la clasificación actual propuesta por la Organización Mundial de la Salud (oms, 2018) para determinar la obesidad (tabla 1), el cual corresponde a la relación entre el peso expresado en kilos y el cuadrado de la altura, expresada en metros. Para determinar el índice de masa corporal, a todos los participantes se les midió el peso y la talla.

Tabla 1

Clasificación de la obesidad según la oms 2018

\begin{tabular}{ccc}
\hline Clasificación & $\begin{array}{c}\text { Personas menores de } 65 \text { años } \\
\text { IMC kg/m2 }\end{array}$ & $\begin{array}{c}\text { Personas mayores de } 65 \text { años } \\
\text { IMC kg/m2 }\end{array}$ \\
Normo peso & $18,5-24,9$ & $21-22,9$ \\
Sobrepeso & $25-29,9$ & $23-28$ \\
Obesidad grado I & $30-34,9$ & $28,1-29,9$ \\
Obesidad grado II & $35-39,9$ & $30-31,9$ \\
Obesidad grado III & $\geq 40$ & $\geq 32$ \\
\hline
\end{tabular}

Al igual que la circunferencia de la cintura (tabla 2), todas las mediciones antropométricas se realizaron tomando en cuenta las consideraciones de la guía para las mediciones físicas de vigilancia de la oms.

\section{Tabla 2}

Clasificación del riesgo de salud cardiovascular según circunferencia de cintura OMS 2018

\begin{tabular}{ccccc}
\hline & & Riesgo bajo & Riesgo moderado & Riesgo alto \\
Hombres & Cintura & $\leq 94 \mathrm{~cm}$ & $94-102 \mathrm{~cm}$ & $\geq 102$ \\
Mujeres & Cintura & $\leq 80 \mathrm{~cm}$ & $80-88 \mathrm{~cm}$ & $\geq 88 \mathrm{~cm}$ \\
\hline
\end{tabular}

Para las determinaciones de los parámetros bioquímicos glucosa, colesterol y triglicéridos, se realizaron por métodos enzimáticos de acuerdo con las instrucciones contenidas en el kit de cada reactivo. Se consideraron valores normales de glucosa menores a $100.0 \mathrm{mg} / \mathrm{dl}$ en ayuno, y valores anormales mayores o iguales a $111.0 \mathrm{mg} / \mathrm{dl}$. En cuanto al colesterol, se consideró como hipercolesterolemia una concentración mayor o igual a 200.0, valores inferiores fueron considerados normales. Para los triglicéridos se consideró normal una concentración inferior a $150.0 \mathrm{mg} / \mathrm{dl}$ y mayores a $200.0 \mathrm{mg} / \mathrm{dl}$ como hipertrigliceridemia. 
Para el procesamiento estadístico de los datos se utilizó el software Estadístico spss en su reciente versión y Microsoft Excel Windows. Se efectuaron tablas de frecuencia expresadas en cantidades. Se efectuaron estadísticas (media aritmética y desviación estándar, valores mínimos y máximos) de cada intervalo, teniendo en cuenta que se ha trabajado con un intervalo de confianza para la media del 95\%, asimismo, se aplicó el test de Chicuadrada para relacionar las variables independientes con las dependientes, considerándose como significativa una $\mathrm{p}<0.05$.

\section{RESULTADOS}

De los 294 estudiantes 107 presentaron sobrepeso (36\%) y 38 (13\%) obesidad tipo I (gráfica 1). Un estudio realizado en España por Arroyo et al., (2006) en estudiantes universitarios, registró una prevalencia de sobrepeso y obesidad de $17,5 \%$ ( $25 \%$ en hombres y $13,9 \%$ en mujeres). De igual manera, un estudio más reciente realizado por Zayas et al., (2014) en estudiantes universitarios de la facultad de medicina en Paraguay, registró una prevalencia de sobrepeso y obesidad de $38,2 \%$. En Chiapas estudios realizados por la Secretaría de Salud del Estado, registraron que los adolescentes de 12 a 19 años presentaron un porcentaje de sobrepeso y obesidad de $28.9 \%$, y para los adultos mayores de 20 años fue de $70.9 \%$ para hombre y $60.6 \%$ para mujeres (Instituto Nacional de Salud Pública, 2013), lo que concuerda con nuestro estudio.

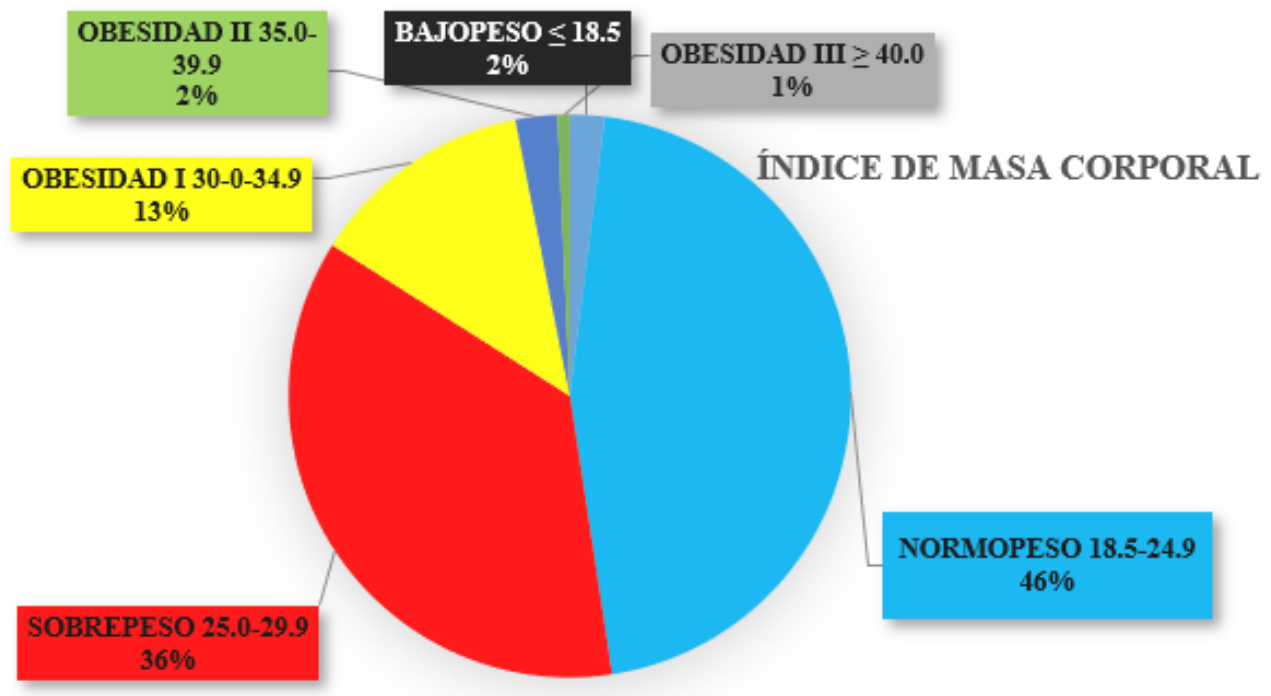

Gráfica 1. Distribución de la población según el IMC 
Con respecto al riesgo para enfermedades cardiovasculares, la distribución de circunferencia de cintura fue de 85 estudiantes (29\%) con riesgo moderado, y 39 (13\%) presentaron riesgo alto (gráfica 2), observándose una tendencia hacia riesgo de salud mayor en las mujeres en comparación con los hombres (gráfica 3). Como afirma Sánchez et al. (2012), las mujeres presentan la tendencia a tener una mayor cc, lo que concuerda con nuestros resultados obtenidos.

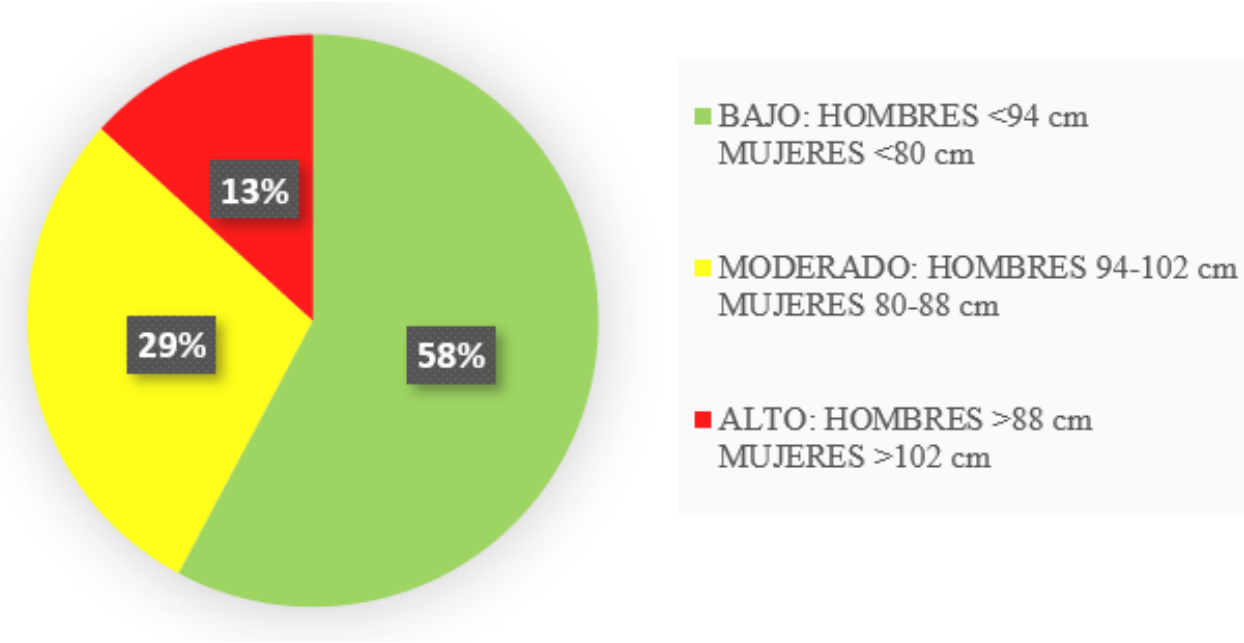

Gráfica 2. Distribución de la población según la CC

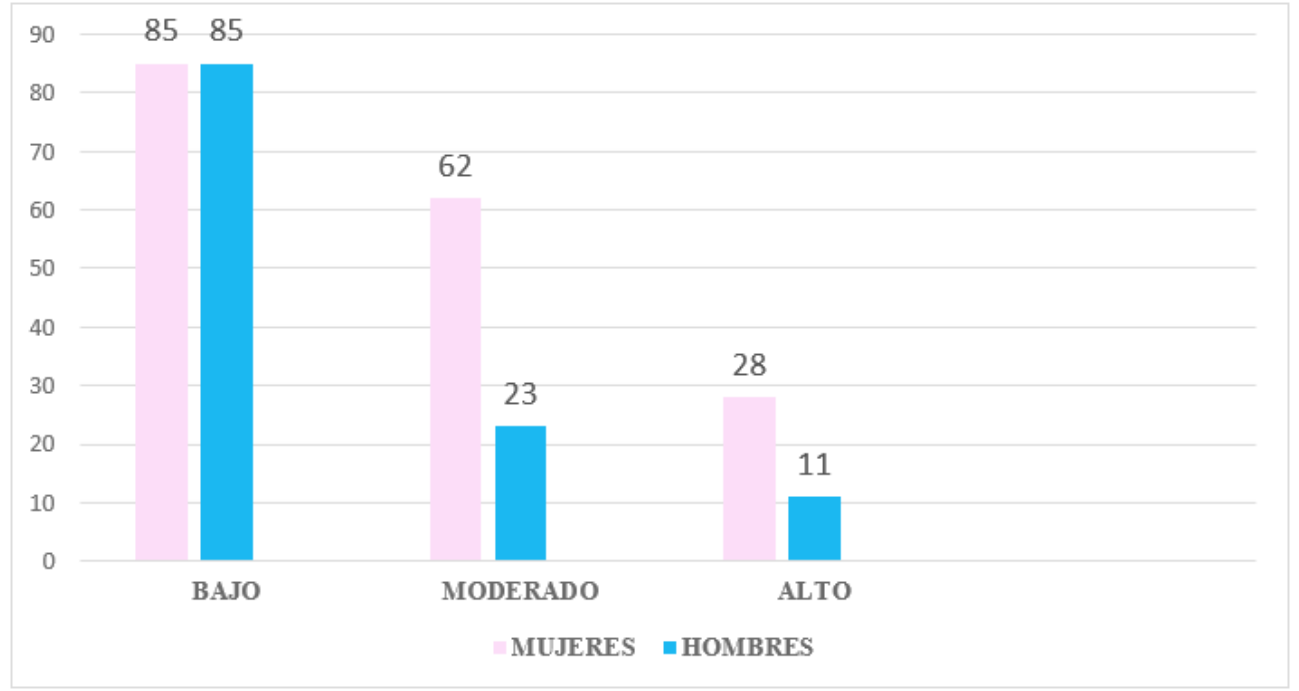

Gráfica 3. Riesgo de salud cardiovascular y sexo

Al analizar el IMC y el parámetro bioquímico glucosa, no se encontró asociación entre estas variables $(\mathrm{p}=0.477)$. Sin embargo, se observó una tendencia en 93 estudiantes (35\%) con valores normales de glucosa que 
tienden al sobrepeso (gráfica 4). Gutiérrez (2009) en un estudio de IMC y su relación con colesterol y triglicéridos no encontró asociación entre el IMC y la glucosa, lo que concuerda con nuestros resultados. Esto probablemente debido a que los estudiantes son muy jóvenes. Sin embargo, la relación entre estas variables debe tomarse en cuenta, ya que, como afirma Argote et al. (2010) los trastornos del metabolismo de los carbohidratos en adolescentes con obesidad, la obesidad y la hiperglicemia constituyen factores de riesgo asociados relevantes.

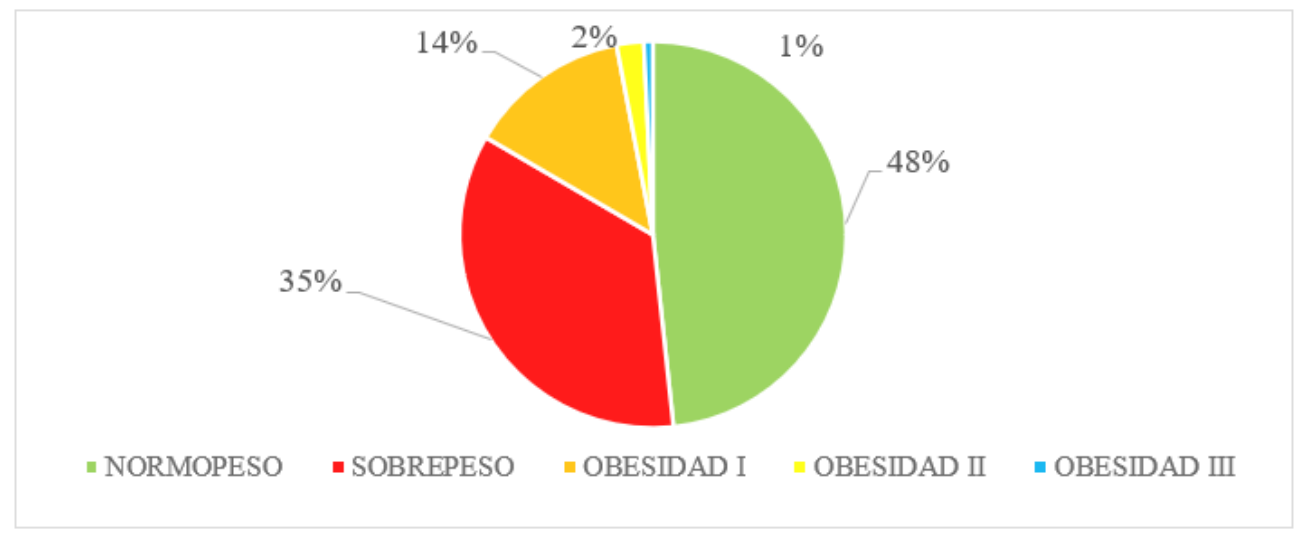

Gráfica 4. IMC y glucosa

Con relación al IMC y el colesterol, no se encontró diferencia estadística significativa entres estas variables $(\mathrm{p}=0.313)$. Sin embargo, se observó una tendencia en 99 estudiantes (37\%) con valores normales, y solo en el $32 \%$ de los casos con valores altos de colesterol que tienden al sobrepeso (gráfica 5). Esto concuerda con un estudio realizado por Jiménez (2011), el cual encontró que las personas con hipercolesterolemia que tienen sobrepeso son 35,2 $\%$ y con obesidad es $27,8 \%$, evidenciando que más personas con sobrepeso sufren de hipercolesterolemia. De igual manera Sam (2011), registró que 32 $\%$ de las personas que acudieron al laboratorio de Ciencias Farmacéuticas tenían IMC elevado e hipercolesterolemia. Asimismo, se puede extrapolar con los datos hallados por Angulo et al. (2009) donde observa que el 35,9\% de niños con obesidad tienen hipercolesterolemia. 


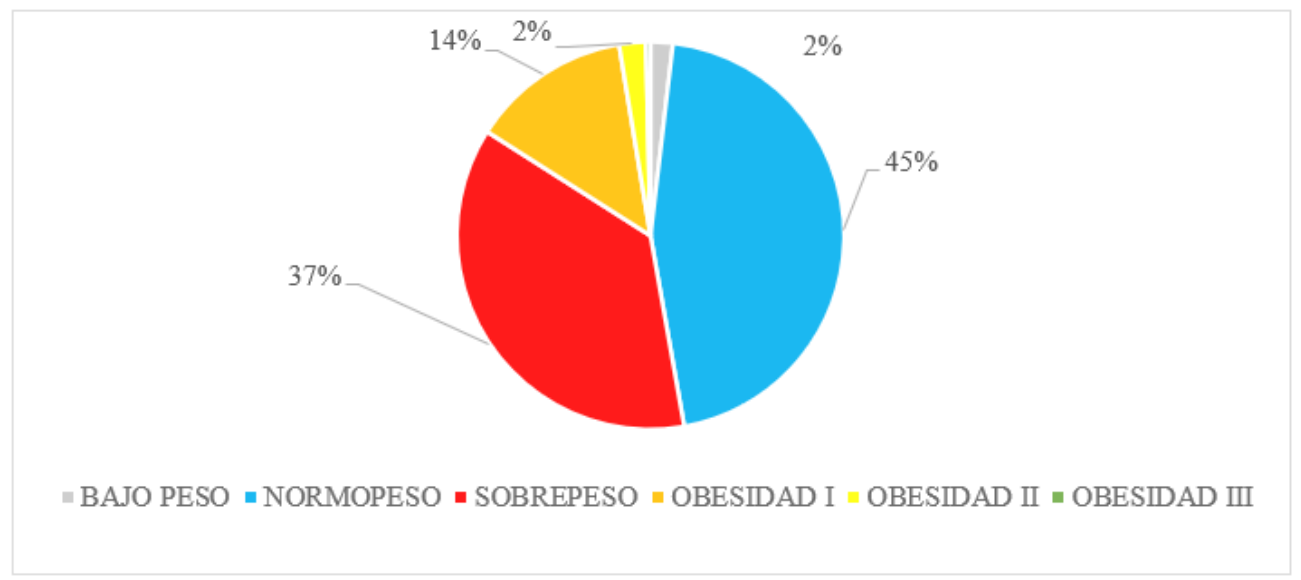

Gráfica 5. IMC y colesterol

Asimismo, con el IMC y los triglicéridos no se encontró relación entre las dos variables $(\mathrm{p}=0.945)$, pero se observó una tendencia en 102 estudiantes (36\%) con valores normales de este parámetro, y en el 50\% de los casos con niveles altos hacia el sobrepeso (gráfica 6). Esto concuerda con lo registrado por Jiménez (2011), en el cual el mayor porcentaje de hipertrigliceridemia $81,7 \%$ los tienen las personas con sobrepeso.

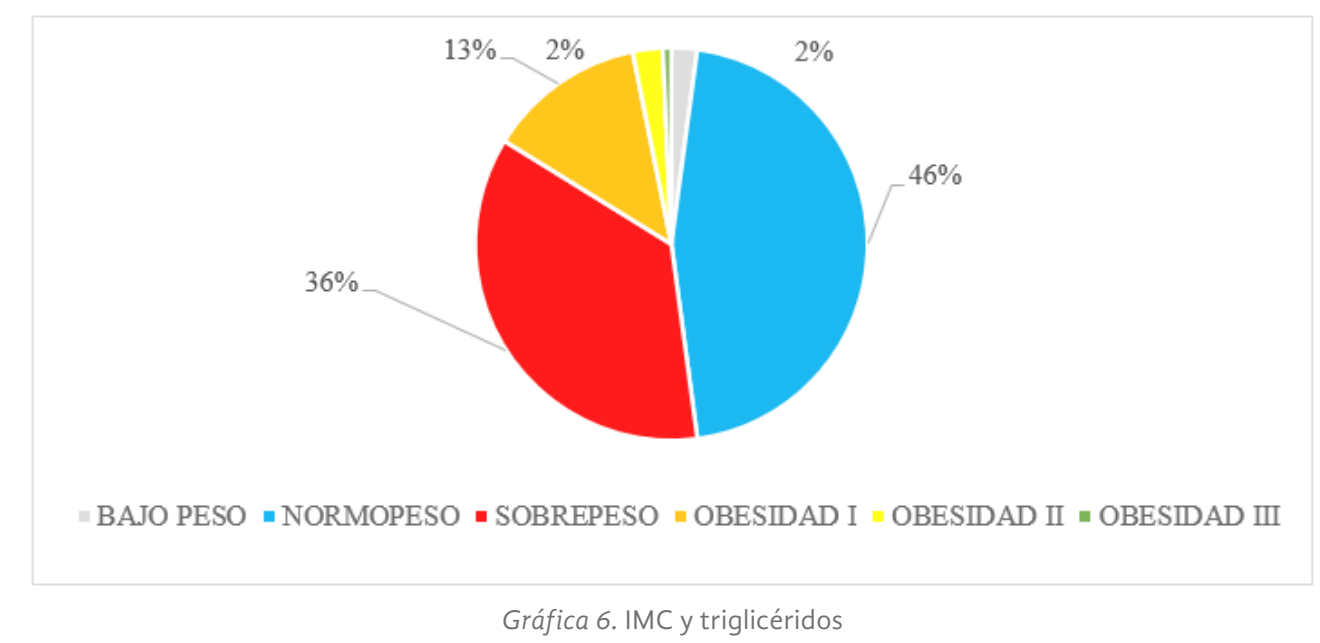

De igual manera, no se encontró relación entre CC y glucosa $(\mathrm{p}=0.059)$, pero existe un alto riesgo de salud en 37 estudiantes (14\%) con niveles de glucosa normal, y solo en un $10 \%$ de los casos con glucosa alta (gráfica 7). Esto difiere con Maceda (2012) en la Evaluación de factores de riesgo en glicemia basal, que la circunferencia de cintura es un factor de riesgo para glicemias elevadas y eventos vasculares cerebrales. Asimismo, Pérez y Díaz (2011) afirman que los individuos con mayor circunferencia de cintura presentan mayores niveles de glucosa en sangre; ellos precisan que esto se debe a una 
insulino resistencia, sin embargo, cabe mencionar que la posible diferencia se puede deber a que estas personas no sufrían de insulino resistencia, debido a que no tenían valores de glicemia tan elevados.

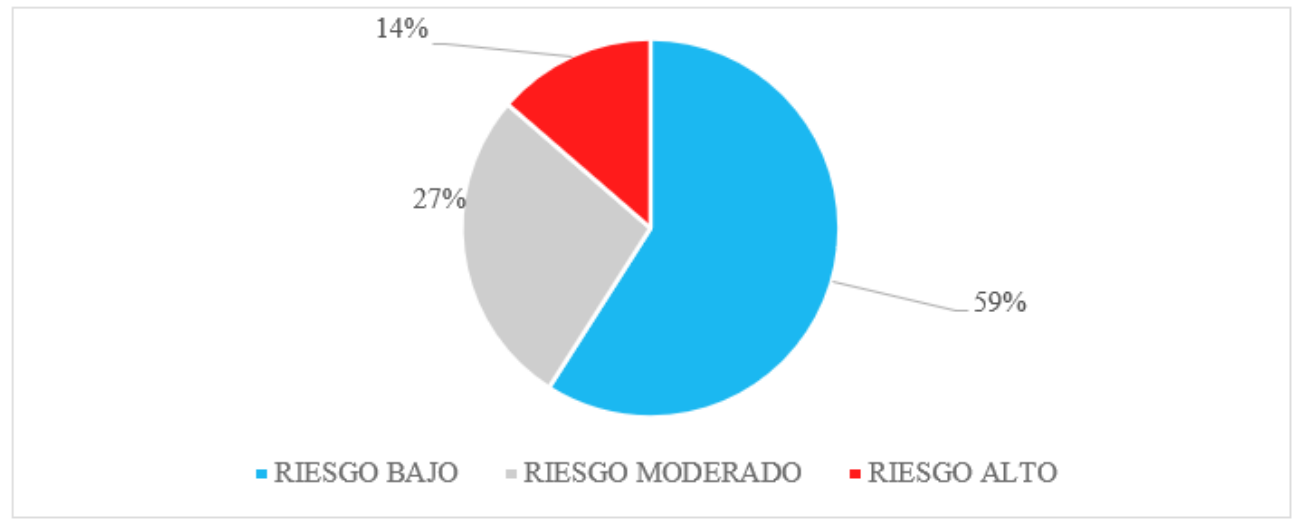

Gráfica 7. CC y glucosa

En cuanto a la cc y el colesterol, si bien tampoco se encontró diferencia estadísticamente significativa $(\mathrm{p}=0.817)$, sí se observó un riesgo de salud alto en 35 estudiantes (13\%) con niveles de colesterol normal, y en el $16 \%$ de los casos con colesterol alto (gráfica 8). Estudios realizados por Rosas et al, Martínez et al, y Pajuelo et al, en el Síndrome Metabólico en adolescentes con sobre peso y obesidad, mencionan la relación entre el aumento de circunferencia de cintura e hipercolesterolemia, trayendo consigo en los últimos años el riesgo de enfermedades cardiovasculares y el síndrome metabólico.

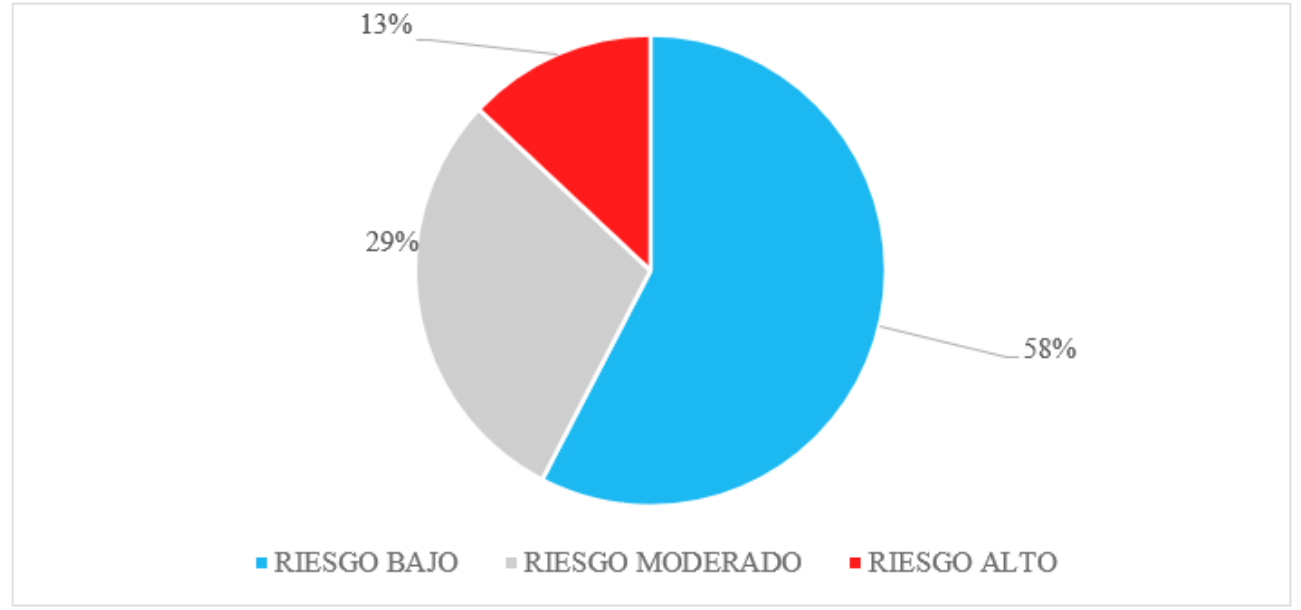

Gráfica 8. CC y colesterol 
Por último, no se encontró correlación entre las variables cc y triglicéridos $(\mathrm{p}=0.402)$, pero se observó un riesgo alto de salud en 39 estudiantes (13\%) con niveles normales de este parámetro bioquímico (gráfica 9), lo cual debe tomarse en cuenta, ya que Pérez (2011) y Pajuelo et al, mencionan que los individuos con mayor circunferencia de cintura presentan mayores niveles de triglicéridos. Asimismo, Sánchez et al, mencionan que los triglicéridos y la circunferencia de cintura son parámetros con mayor precisión para determinar el Síndrome Metabólico, por lo tanto, se podría deducir que un gran porcentaje de esta población se encuentra en riesgo de sufrir este desorden metabólico.

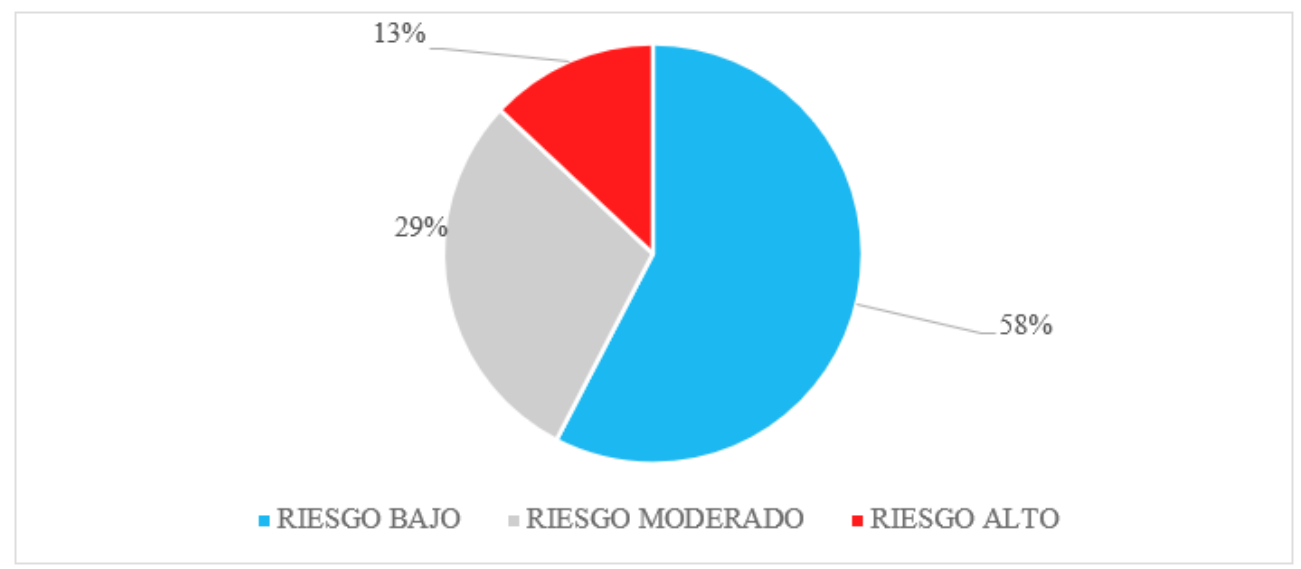

Gráfica 9. CC y triglicéridos

\section{CONCLUSIONES}

En la investigación sobre la relación del índice de masa corporal (IMC) y circunferencia de la cintura (cc) con la glucosa, colesterol y triglicéridos en estudiantes universitarios, se llegó a las siguientes conclusiones:

1. De los 294 estudiantes 107 presentaron sobrepeso (36\%) y 38 (13\%) obesidad tipo I.

2. Con respecto al riesgo para enfermedades cardiovasculares, la distribución de circunferencia de cintura fue de 85 estudiantes (29\%) con riesgo moderado, y 39 (13\%) presentaron riesgo alto, observándose una tendencia hacia riesgo de salud mayor en las mujeres, en comparación con los hombres.

3. No se encontró relación entre el IMC y los parámetros bioquímicos glucosa, colesterol y triglicéridos.

4. No se encontró relación entre cc y los parámetros bioquímicos glucosa, colesterol y triglicéridos 


\section{RECOMENDACIONES}

A pesar de que no hubo resultado estadísticamente significativo entre las variables IMC y CC con los parámetros bioquímicos glucosa, colesterol y triglicéridos, se hace necesaria la detección temprana utilizando indicadores (como el IMC) como medida de prevención para enfermedades como la diabetes mellitus, ya que la prevalencia de diabetes por diagnóstico médico previo en personas de 20 años o más en Chiapas fue de $5.6 \%$, similar a la reportada en la Encuesta Nacional de Salud y Nutrición (2012). Dicha prevalencia de diabetes fue ligeramente mayor en mujeres (6.3\%) que en hombres (4.8\%), con una razón mujer-hombre de 1:3 (Instituto Nacional de Salud Pública, 2013). 


\section{REFERENCIAS}

Angulo N, Zarvas B, Lopez M, Castro de Kolster C. (2009). Índice de Masa Corporal, dislipidemia e hiperglicemia en niños obesos. Comunidad $y$ salud. 2009; 7 (1): 1-8

Argote J, Licea M, Carvajal F, Gonzales R. (2010). Trastornos del metabolismo de los carbohidratos (glucemia en ayunas alterada, tolerancia a la glucosa alterada y diabetes tipo 2) en niños y adolescentes con obesidad. Consulta: agosto del 2019.

Arroyo Izaga, M., Rocandio Pablo, A. M., Ansotegui Alday, 1., Pascual Apalauza, E., Salces Beti, I., y Rebato Ochoa, E., (2006). Calidad de la dieta, sobrepeso y obesidad en estudiantes universitarios. Revista Nutrición Hospitalaria. Universidad del País Vasco.

Gutiérrez E. (2009). Colesterol y triglicéridos y su relación con el índice de masa corporal (IMC) en pacientes adultos que acuden al Servicio Académico Asistencial de Análisis Clínicos (sAAAC). Tesis para optar al Título profesional de Químico Farmacéutico. Facultad de Farmacia y Bioquímica UnMSM; Lima, Perú. 2009.

Instituto Nacional de Salud Pública (2012). Encuesta Nacional de Salud y Nutrición resultados por Entidad Federativa (2012). Chiapas. Cuernavaca, México: Instituto Nacional de Salud Pública, 2013. Disponible en: encuestas.insp.mx

Jiménez D. 2011Relación de Colesterol y Glucosa versus Índice de Masa Corporal en pacientes adultos que acuden al sAAAC (enero-marzo 2010). Tesis para optar al Título profesional de Químico Farmacéutico. Facultad de Farmacia y Bioquímica unMsm; Lima, Perú.

Martínez - Palomino G, Vallejo M, Huesca C, Álvarez de León E, Paredes G, Lerna C. (2006). Factores de Riesgo Cardiovascular en una muestra de mujeres jóvenes mexicanas. Arch Card Mex; 76(4): 401-407

Maceda W, Limo D, col. Evaluación de factores de riesgo en glicemia basal. (2012). Revista peruana de Epidemiologia. (pp. 4) Consulta septiembre del 2019.

Núñez Flores, M. I. (2014). Las Variables: Estructura y Función en la Hipótesis. Investigación Educativa, 11(20), 163-182.

Organización Mundial de la Salud (oms). Nota descriptiva febrero de 2018. Consultada en enero de 2019 y Disponible en: http://www.who.int/ mediacentre/factsheets/fs311/es/

Organización Mundial de la Salud (oms), Guía para las mediciones físicas 2018. Consultada en enero de 2019 y Disponible en: https://www.who.int/ ncds/surveillance/steps/Parte3_Seccion4.pdf

Organización Mundial de la Salud (oms). Informe Mundial sobre la Diabetes (2016). Resumen de orientación (p.6). 
Pajuelo J, Bernui I, Norbelto V, Peña A, Zevillanos L. (2007). Síndrome Metabólico en adolescentes con sobrepeso y obesidad. Anales Facultad Medicina. 68 (2): 133-149

Parreño, T. J. M., y Gutiérrez, P. E., (2010). Colesterol y Triglicéridos y su relación con el índice de Masa Corporal en pacientes adultos en Lima Metropolitana. Revista de Investigación de la Universidad Norbert Wiener. Lima Perú.

Pérez S, Díaz G. (2011). Circunferencia de la Cintura en Adultos, Indicador de Riesgo de Ateroesclerosis. Revista Habanera de Ciencias Médicas. 10 (4): 441- 447

Rosas A, Lama G, Llano F, Dunstan J. (2002). Prevalencia de obesidad e hipercolesterolemia en trabajadores de una institución estatal de Lima - Perú. Revista Perú Médica exp Salud Pública 2002; 19 (2): 87-92

Rivera, R. S., León, B. G. A., Muñetón, P. P., y Silva. C. B., (2013). Obesidad en México: Recomendaciones para una Política de Estado. Epub. Universidad Nacional Autónoma de México. Dirección General de Publicaciones y Fomento Editorial. México, D. F.

Sam S. De la Campaña de Determinación de colesterol y Triglicéridos realizado el 4 de marzo del 2011 y su relación con el índice de masa corporal (IMC) en pacientes adultos que acudieron al laboratorio $\mathrm{N}^{\circ} 05$ de la Facultad Ciencias farmacéuticas y Bioquímica. Consulta: agosto del 2019.

Sánchez J, Pajuelo J, Torres H, Miranda M. (2012). Prevalencia del Síndrome Metabólico en pobladores peruanos por debajo de 1000 y por encima de los 3000 msnm. Anales de la Facultad de Medicina.; 73 (2): 101-106

Tuta-García, H. Y., Lee-Osorno, B. I., Martínez-Torres, J. (2015). Prevalencia $\mathrm{y}$ factores asociados a sobrepeso y obesidad, en estudiantes universitarios de 18 a 25 años, en Pamplona Norte de Santander durante el primer periodo del 2013. Artículo de Investigación Científica. CES Salud Pública 6: 19-24.

Zayas Valdez, V. E., Miranda, C., Scott, C., Viveros, C. (2014). Prevalencia de sobrepeso y obesidad en estudiantes de la Facultad de Medicina de la Universidad Nacional de Itapúa. Revista virtual Soc. Parag. Med. Int. 1(1): 28-36 consultada en: http://dx.doi.org/10.18004/rvsp$\mathrm{mi} / 2312-3893 / 2014.01(01) 28-0362018$. 